\title{
Das Potenzial von vertikalen Windenergieanlagen im Kontext wachsender Flächennutzungskonflikte und Akzeptanzprobleme der Windenergie
}

\author{
Lukas Kern $^{1,4}$ • Johann Valentin Seebaß ${ }^{2,4}$ • Jan Schlüter ${ }^{3,4}$ (D) \\ Online publiziert: 26. November 2019 \\ (c) Der/die Autor(en) 2019
}

\section{Zusammenfassung}

Der Ausbau der Windenergie geht mit zunehmenden Akzeptanzproblemen und unterschiedlichen Flächennutzungskonflikten einher. Diese ergeben sich maßgeblich aufgrund der großen visuellen und akustischen Signatur von horizontalen Windenergieanlagen und dem großen Flächenbedarf von Windparks. Gegenwärtig werden Untersuchungen zu einem Anlagentyp durchgeführt, der die Möglichkeit bietet, diese negativen Effekte deutlich zu reduzieren. Dabei handelt es sich um einen speziellen Anlagentyp mit vertikaler Achsenausrichtung. Diese Anlagenform stand bisher wenig im Fokus der Öffentlichkeit und Wissenschaft stand. Die Vorteile dieser Anlagenform sind ihre niedrige Bauhöhe und ihr geringer akustischer Fußabdruck. Neue Forschungen zeigen zudem, dass die Energiedichte dieser Anlagen diejenige von konventionellen Windenergieanlagen um ein Vielfaches übersteigen kann, wenn man sie in einer Windparkkonfiguration implementiert. Der vorliegende Artikel soll diesen Typ von Windkraftanlage in Bezug auf akzeptanzmindernde Faktoren beleuchten. Vertiefend wird die Schallausbreitung dieser Anlagen anhand einer Schallimissionsprognose untersucht, da es bisher kaum Studien zu dieser Thematik aus raumplanerischer Sicht gibt. Die Ergebnisse zeigen, dass dieser Anlagentyp gegenüber den konventionellen Anlagen neue Flächennutzungsmöglichkeiten bietet und eine Alternative zu den bestehenden Anlagentypen im Rahmen der Energiewende darstellen könnte.

Schlüsselwörter Windenergie · Vertikale Windenergieanlagen · Flächennutzungskonflikte · Akzeptanzprobleme

$\triangle$ Jan Schlüter

jan.schlueter@ds.mpg.de

1 Fakultät für Geowissenschaften und Geographie, Georg-August-Universität, Goldschmidtstraße 3, 37077 Göttingen, Deutschland

2 Lehrstuhl für Statistik, Fakultät für Wirtschaftswissenschaften, Georg-August-Universität, Humboldallee 3, 37073 Göttingen, Deutschland

3 Institut für Dynamik komplexer Systeme, Fakultät für Physik, Georg-August-Universität, Friedrich-Hund-Platz 1, 37073 Göttingen, Deutschland

4 Abteilung Dynamik Komplexer Fluide, Max-Planck-Institut für Dynamik und Selbstorganisation, Am Fassberg 17, 37077 Göttingen, Deutschland 


\title{
The Potential of Vertical Wind Turbines in the Context of Growing Land use Conflicts and Acceptance Problems of the Wind Energy Sector
}

\begin{abstract}
The expansion of wind power is accompanied by a decreasing social acceptance and various land-use conflicts. They are mainly caused by the large acoustic and the large visual footprint of conventional Horizontal Axis Wind Turbines (HAWTs). Currently research is carried out on a wind turbine that provides the capability to avoid these negative effects. It refers to a special type of Vertical Axis Wind Turbines (VAWTs). This wind turbine form hasn't been at the focus of scientific or public discussion so far. The overall advantages are its low installation height and its low noise emissions resulting in a smaller visual and acoustic footprint. Recent research that combined wind energy with bioengineering also showed that the power density of VAWTs can be higher than the power density of HAWTs. By analyzing the existing literature, the potential of these wind turbines is explored in this article. Furthermore the sound propagation of these systems will be examined in more detail on the basis of an emission forecast, as there are few studies on this topic from a regional planning point of view. The results show that VAWTs provide new land-use options compared to the conventional HAWTs and could be an alternative wind turbine type within the energy transition.
\end{abstract}

Keywords Wind energy $\cdot$ Vertical axis wind turbines $\cdot$ Land use conflicts $\cdot$ Acceptance problems

\section{Einführung}

Der verstärkte Ausstoß klimawirksamer Gase ist der Hauptgrund für den heutigen anthropogenen Klimawandel (IPCC 2018). Der Energiesektor ist dabei durch die Verbrennung fossiler Energieträger der größte Emittent (Umweltbundesamt 2018). Aus diesem Grund wurde Ende des letzten Jahrtausends die Energiewende eingeläutet, bei der unter anderem die Erneuerbaren Energien (EE) die fossilen Brennstoffe ersetzen sollen. Doch der Ausbau der EE geht mit zunehmenden Konflikten einher.

Im Bereich der Windenergie, die den größten Anteil der Energieerzeugung aus den EE ausmacht, rufen insbesondere die große visuelle und akustische Signatur von horizontalen Windenergieanlagen durch ihre Bauhöhe und ihre hohen Schallemissionen eine abnehmende gesellschaftliche Akzeptanz hervor (Fachagentur-Windenergie 2019a). Zudem führen die notwendigen Abstände zu Wohn- und Schutzgebieten, die durch das Arten- Bauplanungs- und Immissionsschutzrecht bedingt sind, sowie die Distanzen, die zwischen benachbarten Anlagen bestehen müssen, zu einem enormen Flächenbedarf. Die Abstände zwischen konventionellen Windenergieanlagen, den sogenannten „Horizontal Axis Wind Turbines“ (HAWTs), sind notwendig, um eine durch Turbulenzen induzierte Leistungsreduzierung zu vermeiden (Brownstein et al. 2016; Dabiri 2011).

Das Potenzial, diese negativen Effekte zu vermeiden, bieten spezielle Windenergieanlagen mit vertikaler Achsenausrichtung. Diese Anlagenform stand bisher wenig im Fokus der Öffentlichkeit und Wissenschaft. Die Vorteile dieser sogenannten „Vertical Axis Wind Turbines“ (VAWTs) sind ihre niedrige Bauhöhe und ihr geringer akustischer Fußabdruck. Neue Forschungen zeigen zudem, dass die Energiedichte dieser Anlagen die von HAWTs um das Zehnfache übersteigen kann, wenn man sie in einer optimierten Windparkkonfiguration implementiert (Dabiri 2011, 2014).

Ziel des Artikels ist es, einen Überblick über den aktuellen Forschungsstand hinsichtlich der Konfigurationsoptimierung von VAWT-Windparks zu geben. Zudem soll das Potenzial dieser Anlagenart im Hinblick auf die derzeitige Akzeptanz- und Flächennutzungsproblematik durch eine Abschätzung der Lärmemissionen einer VAWT im Vergleich zu einer HAWT beleuchtet werden. Da bisher keine derartigen Untersuchungen durchgeführt wurden, kann diese Arbeit als Grundlage für die Planung zukünftiger VAWTWindparks dienen.

Der Artikel ist wie folgt aufgebaut: Nachdem zu Beginn der Flächenbedarf von Windparks erläutert wird, werden die VAWTs näher betrachtet und ihre Vor- und Nachteile im Vergleich zu konventionellen Windenergieanlagen aufgezeigt. Zudem wird der Prozess dargelegt, der bewirkt, dass VAWTs eine höhere Energiedichte erzielen können als HAWTs. Dabei geht es um die Nutzung spezifischer Strömungseigenschaften der VAWTs in einem Array, die es ermöglicht, die Abstände zwischen den Anlagen im Vergleich zu den HAWTs zu verkleinern und somit den Flächenverbrauch zu reduzieren. Anschließend wird auf die akustische Signatur von Windkraftanlagen, insbesondere den der VAWTs, eingegangen. Bei ihnen ist der akustische Fußabdruck aufgrund der niedrigeren Anlagenhöhe und der kleineren Grundlautstärke sowie bestimmten Ausbreitungseigenschaften des Schalls geringer. Im letzten Abschnitt wird anhand einer Schallimissionsprognose gezeigt, wie sich die Ausbreitung des Schalls zwischen HAWTs und VAWTs auf eine bestimmte Entfernung unterscheidet. Dabei wird auch untersucht, wie groß der Abstand zu einem Wohngebiet sein müsste, damit die Anlagen genehmigungsfähig sind. 


\subsection{Flächenverbrauch und -bedarf von Windparks}

Windparks besitzen einen hohen Flächenfußabdruck, da bestimmte Abstände zwischen benachbarten Windenergieanlagen notwendig sind. Der Hauptgrund für diesen Abstand ist, dass Anlagen, die als erstes vom Wind angeströmt werden, Turbulenzen verursachen, die bei stromabwärts liegenden Anlagen zu Energieverlusten führen (Dabiri 2011). Des Weiteren dienen Distanzen dazu, die Ermüdungsbelastungen die auf die Anlagen wirken, zu reduzieren und damit die Haltbarkeit der Anlagen zu verlängern (Hezaveh et al. 2018). Damit die entstehenden aerodynamischen Interferenzen komplett vermieden werden können, müsste der Abstand zwischen zwei HAWT-Anlagen nach Brownstein et al. (2016) ca. das zwanzigfache des Rotordurchmessers der Anlage betragen. In den existierenden Windparks beträgt dieser Abstand hingegen im Durchschnitt nur den drei- bis fünffachen Rotordurchmesser in Nebenwindrichtung und den sechs- bis zehnfachen Rotordurchmesser in Hauptwindrichtung. Da die Rotorblätter in der Regel eine Länge von über $40 \mathrm{~m}$ haben, ergibt sich selbst bei diesen Abständen nach wie vor ein hoher Flächenbedarf (Brownstein et al. 2016).

Neben den Distanzen zwischen benachbarten Anlagen spielen auch die Abstände zu Wohn- oder Schutzgebieten eine Rolle bei der Flächenbedarfsplanung. Sie sind durch das Arten-, Bauplanungs- und Immissionsschutzrecht bedingt und maßgeblich auf die große visuelle und akustische Signatur von HAWTs zurückzuführen. Einerseits müssen bestimmte Abstände aufgrund der landschaftseingreifenden und der optisch bedrängenden Wirkung dieser Anlagen eingehalten werden. Weiterhin ergeben sich die Distanzen aus dem großen akustischen Fußabruck dieser Anlagen. Neben den Auswirkungen auf die Flächennutzung sind die Schallemissionen einer der größten Faktoren für den gesellschaftlichen Akzeptanzverlust von Windenergieanlagen (Hübner and Pohl 2015). Die vergleichsweise hohe Grundlautstärke der Anlagen führt dazu, dass diese noch in relativ großer Entfernung zu hören sind. Auch wenn diese Thematik kontrovers diskutiert wird, gehen viele Wissenschaftler davon aus, dass die Schallemissionen bei Menschen, die in der Umgebung einer Anlage wohnen, auch bei größeren Entfernungen unter anderem zu Kopfschmerzen und Schlafstörungen führen können (LfU 2018; Twardella 2013). Um diese Effekte zu vermeiden und die gesellschaftlichen Bedenken zu reduzieren, werden bestimmte Abstände zwischen den Anlagen und Wohngebieten eingehalten. Die Abstandsempfehlungen zur Ausweisung von Windenergiegebieten zu Wohngebieten liegen in den verschiedenen Bundesländern zwischen $400 \mathrm{~m}$ und $1100 \mathrm{~m}$ (FachagenturWindenergie 2019b).

Auf Basis des sogenannten Flächenbedarfswertes, der angibt, wie viel Hektar pro Megawatt (Ha/MW) an Flä- che benötigt werden, kann man den momentanen Flächenverbrauch von HAWTs in Deutschland abschätzen. Ausgehend von einem durchschnittlichen Flächenbedarfswert von 7 ha/MW (Dena 2010) und einer Gesamtleistung der Onshore-Windenergie von 52,931 MW (BWE 2019a) betrug der Flächenverbrauch Ende 2018 ca. 3700 km². Damit entspräche der Flächenfußabdruck der HAWTs einer Größe von mehr als $1 \%$ der deutschen Landesfläche. Einer Untersuchung der Klimaschutzagentur Hannover nach, spielte die Länge der Rotorblätter bei den Berechnungen des Flächenbedarfswertes bisher kaum eine Rolle. Dieser könnte unter Berücksichtigung der Rotorlänge um 21,5-24\% ansteigen und somit wäre der Flächenverbrauch als noch höher einzuschätzen (DEWI 2015).

Abbildung 1 zeigt die Entwicklung der installierten Gesamtleistung der Windenergie seit der Jahrtausendwende und mehrere Ausbauszenarien. Das Ausbauziel des aktuellen Klimaschutzplans sieht eine erhöhte Gesamtleistung der installierten Onshore-Windanlagen von 69,600 MW bis zum Jahr 2030 vor (Fraunhofer 2019). Damit dieses Ziel erreicht werden kann, werden künftig weiterhin große Flächen benötigt. Um den Flächenverbrauch zu reduzieren, kam es in den letzten Jahren zu einer wachsenden Verdichtung der Windenergieanlagen innerhalb von Windparks (DEWI 2015). Allerdings steigen dadurch die Turbulenzinteraktionen zwischen den verschiedenen Anlagen, was zu einer Abnahme des Nutzungsgrades pro Anlage führt. Daher ist es notwendig, alternative Anlagentypen wie die VAWTs, in Betracht zu ziehen.

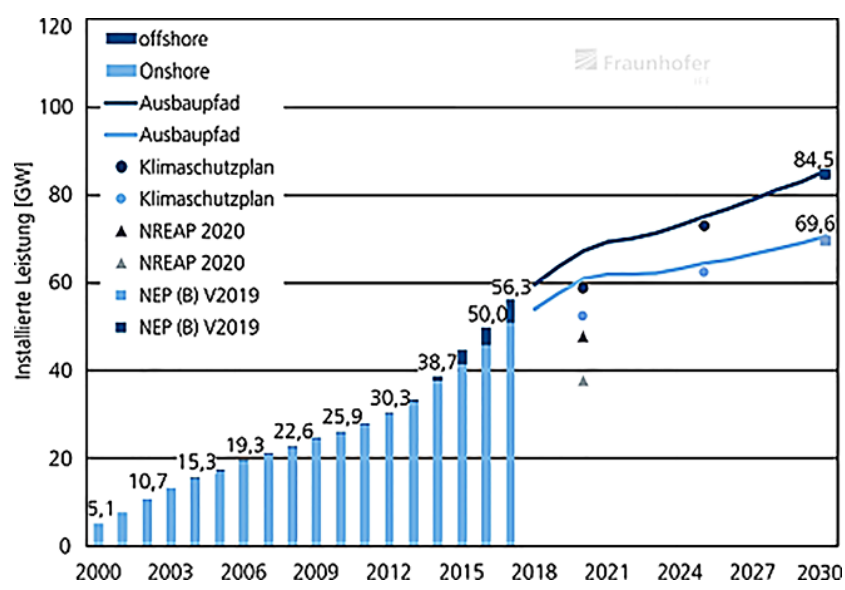

Abb. 1 Entwicklung und Ausbauszenarien der Windenergie (übernommen aus Fraunhofer 2019 mit der Erlaubnis des Fraunhofer IEE). Die Grafik zeigt die bisherige Entwicklung der installierten Windenergieleistung seit 2000 und verschiedene Ausbauszenarien des Klimaschutzplans, des National Renewable Energy Action Plan (NREAP) und des Netzentwicklungsplans Strom (NEP) 


\section{Vertical Axis Wind Turbines}

VAWTs sind eine spezielle Form der Windenergieanlagen, die im Gegensatz zu den konventionellen HAWTs durch eine Rotorachse in vertikaler Lage gekennzeichnet sind. Der Vertikalrotor war die erste Turbinenform, mit der Energie erzeugt wurde. Bereits $900 \mathrm{n}$. Chr. wurden VAWTs von den Persern als Windmühlen für mechanische Arbeiten verwendet (Eriksson et al. 2008). Seit Ende des 19. Jahrhunderts werden Windenergieanlagen zur Stromerzeugung genutzt. Im Zuge dessen wurde erkannt, dass HAWTs höhere Wirkungsgrade besitzen, was ein abnehmendes Interesse an VAWTs zur Folge hatte. Es gibt grundsätzlich zwei verschiedene Arten der VAWT welche aktuell zur Stromerzeugung genutzt werden: den Savonius-Rotor und den Darrieus-Rotor (vgl. Abb. 2). Diese Arbeit beschäftigt sich mit einer speziellen Form des Darrieus-Rotors, dem sogenannten H-Rotor, der in Abb. 4 abgebildet ist. Wie HAWTs, nutzt diese Anlagenform das Auftriebsprinzip zur Energiegewinnung. Im Gegensatz zum klassischen Darrieus-Rotor, der gewölbte Rotorblätter besitzt, hat ein H-Rotor gerade
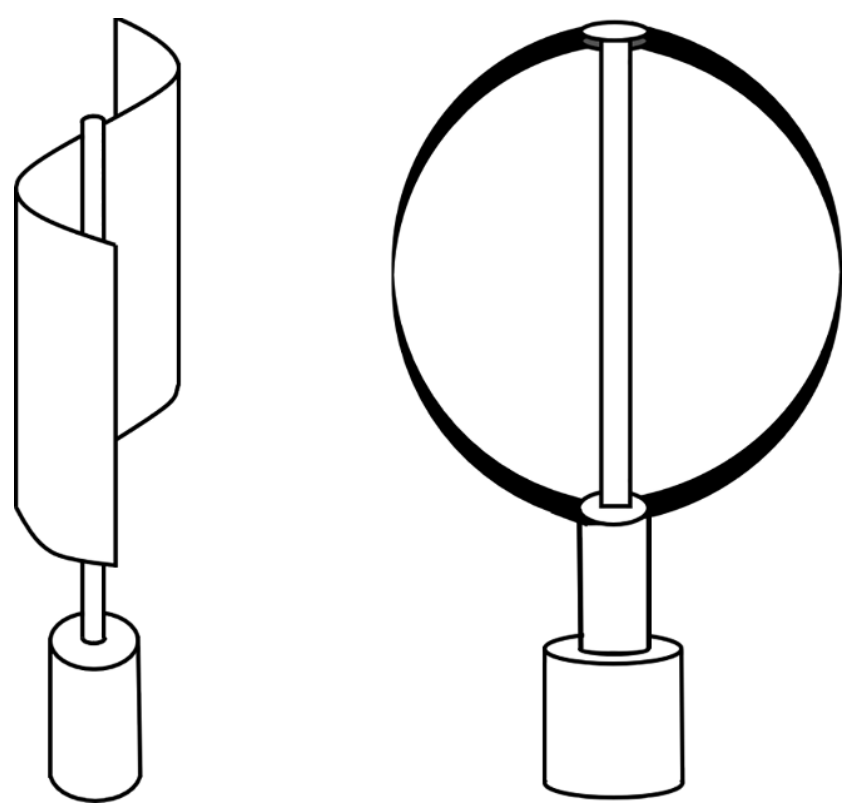

Abb. 2 VAWT-Arten (eigene Darstellung). In dieser Grafik sind die zwei bekanntesten Arten der VAWT, die aktuell zur Stromerzeugung genutzt werden, zu erkennen. Auf der linken Seite ist ein Savonius-Rotor und auf der rechten Seite ein Darrieus-Rotor skizziert. Der Savonius-Rotor verwendet größtenteils den widerstandsbedingten Vortrieb als Wirkungsweise zur Energieerzeugung und wird daher auch als Widerstandsläufer bezeichnet. Der Darrieus-Rotor wird auch als Auftriebsläufer bezeichnet und nutzt durch seine tragflächenförmigen, gebogenen Rotorblätter, wie die konventionellen HAWTs, das Auftriebsprinzip zur Energiegewinnung. Grundsätzlich ist der Leistungsbeiwert, der angibt, wie effizient eine Windenergieanlage Energie in Nutzleistung umwandeln kann, bei Savonius-Rotoren niedriger als bei Darrieus-Rotoren. Der H-Rotor, mit dem sich diese Arbeit befasst, ist eine veränderte Form des Darrieus-Rotors und ist in Abb. 4 zu sehen

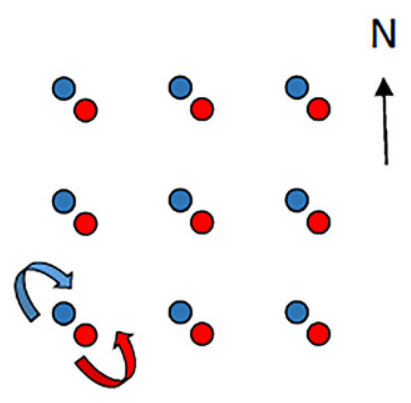

Abb. 3 Skizze des Testfeldes (Eigene Darstellung). Rote Kreise kennzeichnen gegen den Uhrzeigersinn rotierende Turbinen, blaue Kreise mit dem Uhrzeigersinn rotierende Turbinen. Der Abstand zwischen den Turbinen innerhalb eines Paares beträgt das 1,65fache des Turbinendurchmessers (D) $(\mathrm{D}=1,2 \mathrm{~m})$. Die Turbinenpaare weisen untereinander einen Abstand von 8D auf. Der Pfeil gibt die Hauptwindrichtung an

Rotorblätter (Eriksson et al. 2008). H-Rotoren und andere aktuelle vertikale Windkraftanlagen sind in der Regel deutlich kleiner als HAWTs. Bei dem H-Rotor „Windspire 1,2kW turbine" (Windspire 2019), um den es in diesem Artikel hauptsächlich geht, beträgt die Bauhöhe 9,1 m, während heutige HAWTs im Durchschnitt $128 \mathrm{~m}$ hoch sind

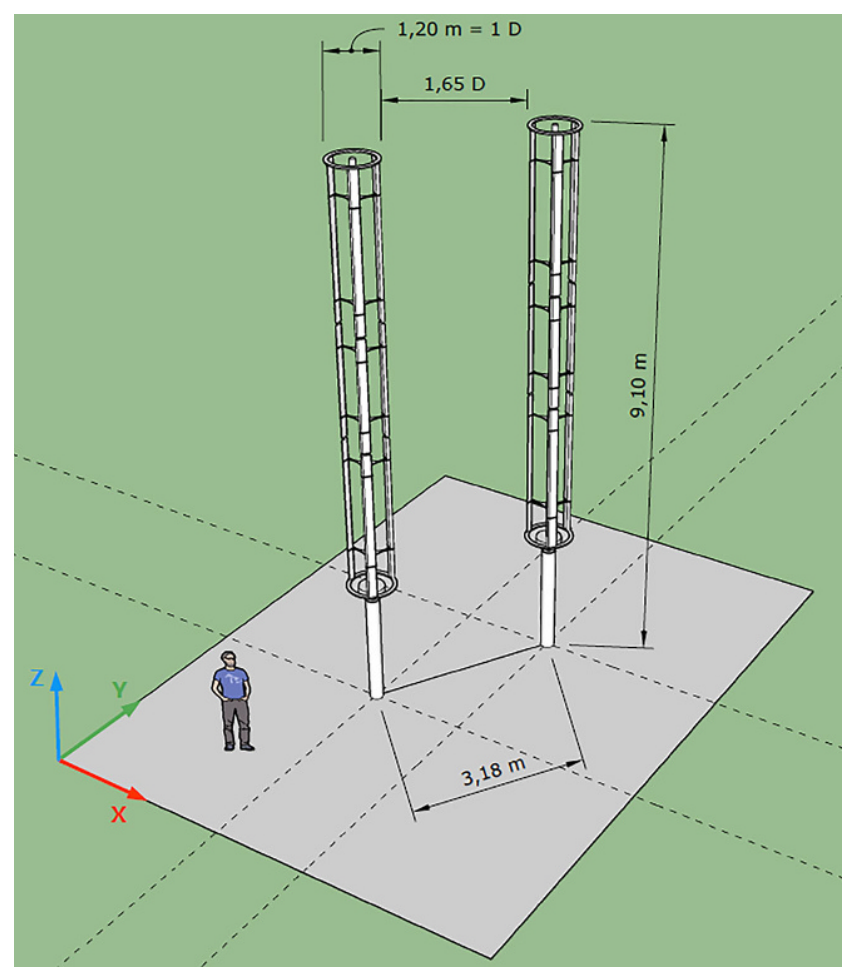

Abb. 4 Skizze eines VAWT-Paares (Eigene Darstellung). Dieses Abbildung zeigt zwei H-Rotoren. Die Rotorenform und die Maße der Anlagen entsprechen denen des Typs „Windspire 1,2kW turbine“ (Windspire 2019). Die Bauhöhe der Turbinen liegt bei 9,1 m. Im Bild ist eine etwa $1,80 \mathrm{~m}$ große Person neben einer Turbine zum Größenvergleich zu sehen. Die Abstände zwischen den einzelnen Turbinen innerhalb eines Paares stimmen mit den Werten aus Abb. 3 überein. Der Pfeil der $Y$-Achse gibt die Hauptwindrichtung an 


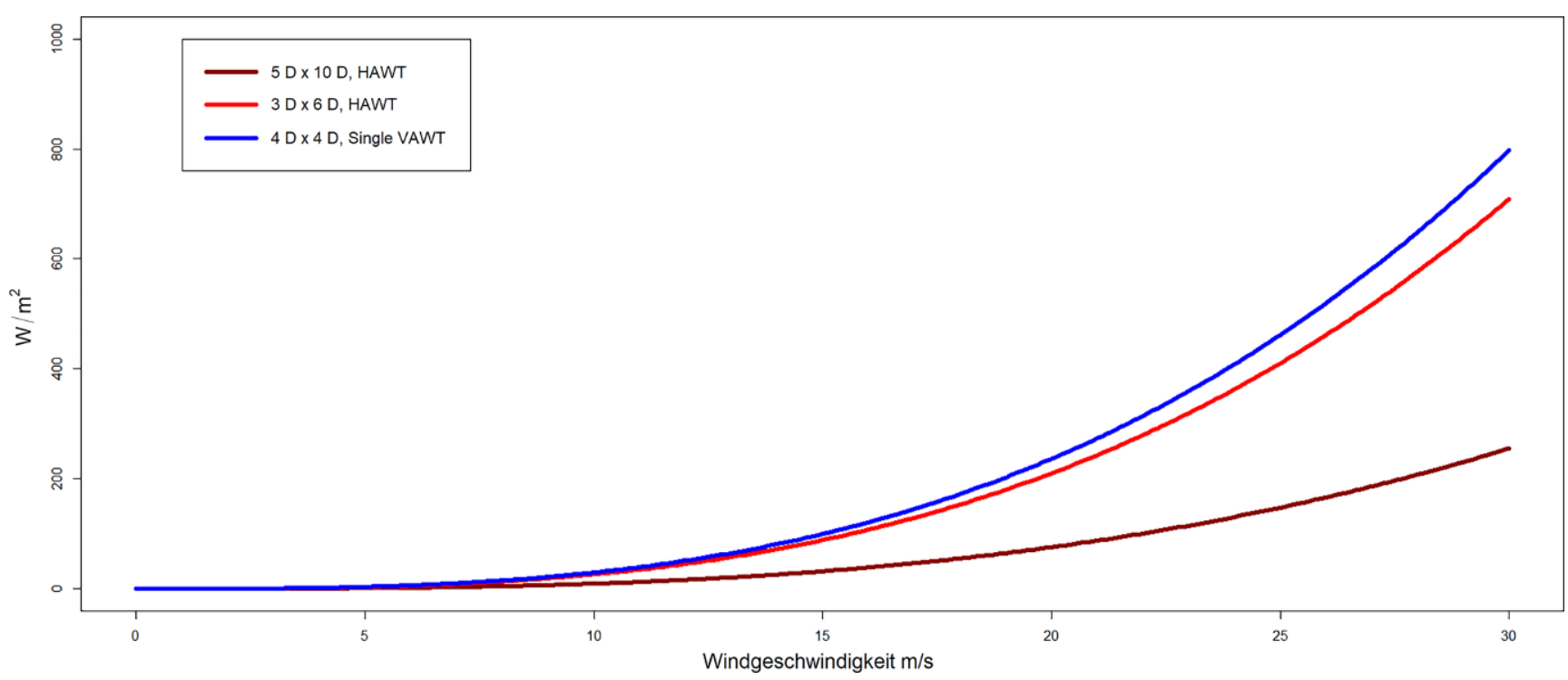

Abb. 5 Vergleich der Energiedichten einer HAWT und einer VAWT (Eigene Darstellung). In dieser Abbildung sind die Energiedichten (W/m²) bei Windgeschwindigkeiten zwischen 0-30 m/s für drei verschiedene Windkraftanlagennachläufe nach dem Betzschen Gesetz berechnet worden (Betz 1920). Aus den Kalkulationen ergibt sich die potenzielle Leistung der jeweiligen betrachteten Fläche $\left(D_{\text {seitliche Richtung }} * D_{\text {Längsrichtung }}\right)$ pro $m^{2}$ bei einer bestimmten Windgeschwindigkeit. Die blaue Linie zeigt die Energiedichte für eine VAWT. In dieser Konfiguration beträgt der theoretische Nachlauf das vierfache des Rotordurchmessers $\mathrm{D}(\mathrm{D}=1,2 \mathrm{~m})$ in seitliche Richtung und 4D in Längrichtung. Die roten Linien beschreiben die Energiedichten für zwei unterschiedliche HAWT-Konfigurationen welche in der Realität häufig existieren. Für die Berechnung der dunkelroten Linie wurde ein Wert von 5D $(\mathrm{D}=112 \mathrm{~m})$ in seitlicher Richtung und 10D in Längsrichtung als Nachlauflänge gewählt. Dieses Konzept weist die niedrigste Energiedichte auf. Für die Kalkulation der hellroten Linie wurden ein Nachlauf von 3D (seitliche Richtung) $(\mathrm{D}=112 \mathrm{~m}) \mathrm{und} 6 \mathrm{D}$ (Längsrichtung) gewählt. Es ist zu erkennen, dass die VAWT-Konfiguration die höchste Energiedichte aufweist

(Weston 2018). Die geringere Anlagenhöhe hat zur Folge, dass der visuelle Fußabdruck deutlich kleiner ist. Die Anlagen sind aus größeren Entfernungen nicht mehr wahrnehmbar und es besteht keine optisch bedrängende Wirkung, die bei HAWTs häufig auftritt (VG Stuttgart 2013). Zudem spielt die Belästigung durch den Schattenwurf bei VAWTs eine untergeordnete Rolle (Hui et al. 2018). Studien zeigen außerdem, dass VAWTs aufgrund ihrer Bauhöhe und der kleineren Rotationsgeschwindigkeit Vorteile hinsichtlich des Vogelschutzes bieten und sie theoretisch in sensibleren Gebieten implementiert werden könnten (Dabiri 2011; Dabiri et al. 2015; Hui et al. 2018). Des Weiteren stellt auch der kleinere akustische Fußabdruck von VAWTs, auf den im dritten und vierten Teil des Artikels näher eingegangen wird, einen wesentlichen Vorteil dieses Windkraftanlagentyps dar.

Dem entgegen steht die geringe Nennleistung dieser VAWT-Anlagen, die sich meist im einstelligen Bereich befindet, sowie ihr geringer Wirkungsgrad als Nachteil. Dies sind die Hauptgründe dafür, weshalb dieser Anlagentyp bisher kaum im Fokus der Öffentlichkeit und der Forschung stand (Eriksson et al. 2008; Islam et al. 2013).

\subsection{VAWTs in einer Windparkkonfiguration}

Prof. John Dabiri vom California Institute of Technology stellte 2009 in einer Computersimulation dar, wie es mög- lich ist, die VAWTs in einem kleinen Array so zu konfigurieren, dass im Vergleich zu den kommerziellen Windparks, zehnmal mehr Energie pro Flächeneinheit produziert werden kann. Der wesentliche Faktor für die höhere Energiedichte ist, dass VAWTs im Vergleich zu HAWTs in einem Windpark deutlich dichter gestaffelt sein können. Trotz sehr geringer Distanzen zwischen benachbarten Anlagen im einstelligen Meterbereich, weisen VAWTs in einer bestimmten Anordnung keine Energieverluste auf. Das Konzept wurde vom Strömungsverhalten der Fischschwärme adaptiert. Wenn sich Fische in einem Schwarm bewegen, bilden sie eine bestimmte Formation, die es dem einzelnen Tier erlaubt, sich vom Wirbel der beiden vorausschwimmenden Fische voranziehen zu lassen (Dabiri 2017; Gemmell et al. 2016; Islam et al. 2013). Der Grund dafür ist die sogenannte konstruktive Interferenz zwischen den hydrodynamischen Wirbeln benachbarter Fische. Die Simulation weist einen besonders hohen Wirkungsgrad auf, wenn die VAWTs als Paare aufgestellt sind, bei denen sich ein Rotor einer Anlage mit dem Uhrzeigersinn und ein Rotor gegen Uhrzeigersinn dreht. So entstehen Verwirbelungen, die denen der beschriebenen Fischschwärme ähneln. Die höhere Effizienz erklärt sich aus der Bewegung des jeweils nächsten Rotors, durch den ein größerer Luftstrom verursacht wird, der von VAWT- Paaren synergetisch genutzt werden kann (Dabiri 2011; Brownstein et al. 2016). In Abb. 3 ist das Konzept eines Testfeldes von Dabiri als Skizze gezeigt. Dort sind 


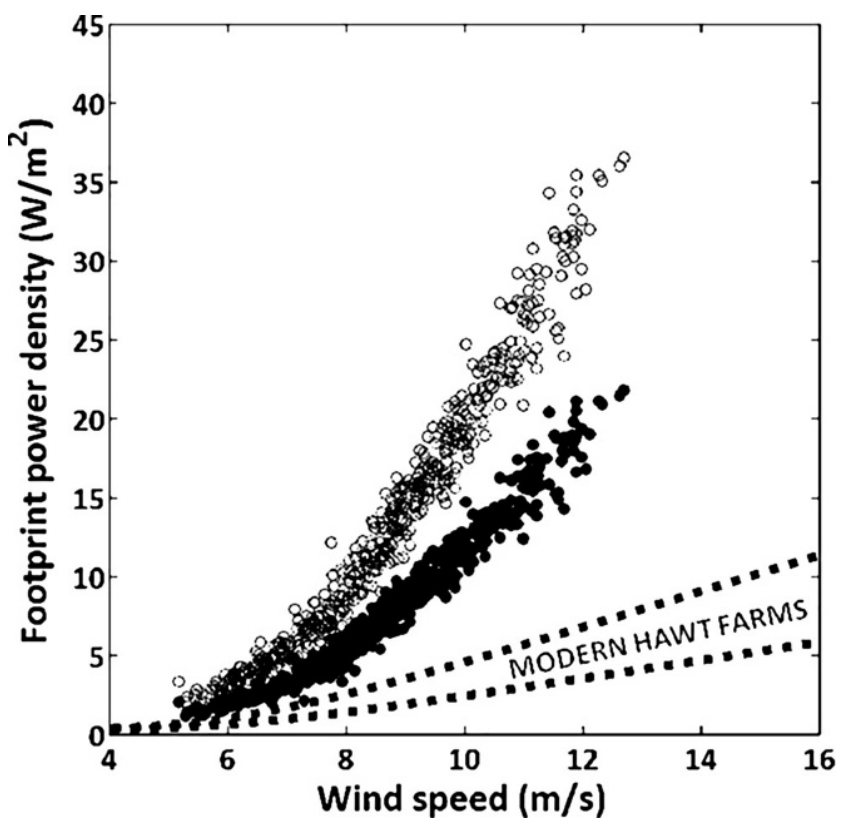

Abb. 6 Vergleich der Energiedichten von HAWTs und VAWTs (Reproduced from Dabiri 2014, with the permission of the American Institute of Physics). Messungen der Energiedichte für einen VAWTWindpark mit 18 Anlagen. Ungefüllte Punkte zeigen direkte Messungen an. Gefüllte Punkte zeigen die Extrapolation der Messungen für ein theoretisches unendlich großes VAWT-Array unter Verwendung eines geometrischen Korrekturfaktors an. Jeder Datenpunkt steht für eine zehnminütigen Durchschnitt der gemessenen Windgeschwindigkeit. Die gepunkteten Linien geben die Spannweite der Performance von modernen HAWT-Windparks an

die Rotationsrichtungen der einzelnen Anlagen innerhalb der Turbinenpaare erkennbar.

Das in Abb. 3 skizzierte VAWT-Testfeld wurde in der kalifornischen Wüste bereits in der Praxis getestet (Kinzel et al. 2015; Dabiri et al. 2015). Eines dieser Anlagenpaare ist in Abb. 4 als Skizze dargestellt.

In dem Array gibt es hauptsächlich zwei strömungsdynamische Mechanismen die gegeneinander wirken und die zur Gesamtperformance beitragen: Erstens die Blockierung der Turbinen („Turbine Blockage“), bei der die Strömung von einer Anlage blockiert und zur benachbarten Anlage umgeleitet wird. Dadurch kann die Leistung des Anlagenpaares höher sein als bei zwei einzelnen, isolierten Anlagen desselben Typs. Zweitens der Nachlauf-Effekt (,Turbine Wakes"), bei dem die Luftströmung lokal verlangsamt wird und der die Leistung im Gegensatz zur „Turbine Blockage“ eher verschlechtert (Dabiri 2014). Dieser Effekt führt bei HAWTs dazu, dass relativ große Abstände zwischen benachbarten Anlagen bestehen müssen, um Energieverluste zu vermeiden. Bei VAWTs wirken sich die „Turbine Wakes“ wesentlich geringer auf die Leistung aus. Aus diesem Grund können VAWTs in einem Array verhältnismäßig dicht angeordnet sein, ohne dass es zu signifikanten Energieverlusten kommt (Dabiri et al. 2015).
Kinzel et al. haben 2015 mithilfe von Versuchen auf einer Testfläche unter realen Windbedingungen die horizontalen und vertikalen Turbulenzen in drei unterschiedlichen Windparkkonfigurationen von VAWTs untersucht. Sie konnten feststellen, dass bei größeren Arrays eine neue Oberflächenschicht (,surface layer flow“) oberhalb der Anlagen entsteht. Die daraus resultierenden Verwirbelungen weisen starke Ähnlichkeiten zum Strömungsverhalten auf, das man von Waldbeständen kennt. Aus diesem Forschungsbereich könnten wichtige Erkenntnisse für VAWTs gewonnen werden. Sie gehen davon aus, dass eine dichte Staffelung der Anlagen zu einem Anstieg des turbulenten Energieflusses über der gesamten Arrayfläche führen könnte, welcher von den VAWTs genutzt werden kann (Kinzel et al. 2015).

Rolin und Porté-Agel entdeckten 2017 zudem, dass im nahen Windschatten einer Anlage zwei in entgegengesetzter Richtung drehende Wirbel entstehen, die zu einer Seitenwindbewegung (,,crosswind motion“) führen. Diese Seitenwinde sind an der energetischen Wiederaufladung stromabwärts der Anlagen beteiligt (Rolin and Porté-Agel 2018).

Da diese Prozesse bereits in unmittelbarer Nähe der VAWTs stattfinden, können diese sehr dicht hinter- und nebeneinander aufgestellt werden, ohne dass es zu Energieverlusten kommt. Computersimulationen und Feldversuche zeigten, dass in einem Array mit VAWTs mehr Energie pro Flächeneinheit erzeugt werden kann als bei Windparks mit konventionellen HAWTs. Die Energiedichte von VAWTs kann dadurch laut Untersuchungen zehn Mal so hoch sein wie die von HAWTs (Dabiri 2011; Dabiri 2014).

Ein wesentlicher Grund dafür ist, dass HAWTs sehr weit von einander entfernt aufgestellt sein müssen. Dadurch resultieren Flächen, in denen die vorhandene kinetische Energie nicht genutzt werden kann. Diese ungenutzten Bereiche entstehen bei VAWTs nicht, da sie sehr dicht hinterund nebeneinander gestaffelt werden können. (Dabiri 2014; Dabiri et al. 2015; Ryan et al. 2016). Abbildung 5 zeigt den Vergleich der Energiedichten in Abhängigkeit von der Windgeschwindigkeit einer HAWT (rote Linien) mit unterschiedlichen Nachlaufkonfigurationen in Relation zur Rotorgröße (D) und einer VAWT (blaue Linie). Anhand dieser Kalkulationen ${ }^{1}$ nach Betz ist eine höhere Energiedichte bei der VAWT als bei gängigen HAWTs zu erkennen (Betz 1920). In den Berechnungen von Dabiri, die in Abb. 6 zu sehen sind, ist der Unterschied der Energiedichte zwischen

\footnotetext{
1 Verwendetes Rechenmodell:

$P d=c_{\mathrm{p}} * \rho * D * v^{3} /(D * S)$

$P d=$ Energiedichte

$c_{\mathrm{p}}$ (Leistungsbeiwert) $=0,5$

$\rho$ (Luftdichte) $=1,204 \mathrm{~kg}$ pro $\mathrm{m}^{3}$

$D$ (Durchmesser) $=1,2 \mathrm{~m}$ (VAWT) bzw. $112 \mathrm{~m}$ (HAWT)

$v$ (Windgeschwindigkeit) $=0-30 \mathrm{~m} / \mathrm{s}$

$S$ (Abstände der jeweiligen Nachlaufkonfiguration) $=4 * 4$ (VAWT), $3 * 6\left(\mathrm{HAWT}_{1}\right), 5 * 10\left(\mathrm{HAWT}_{2}\right)$
} 
Abb. 7 Skizze eines Windparks mit triangulären Clustern (eigene Darstellung). Schematische Übersicht der VAWT Windparkkonfiguration mit triangulären Clustern die von Hezaveh et al. 2018 anhand von Computersimulationen getestet wurden. Der Abstand zwischen den Turbinen innerhalb eines Clusters beträgt jeweils 4D $(\mathrm{D}=1,2 \mathrm{~m})$. Der Abstand zwischen zwei Clustern beträgt in seitlicher Richtung (X-Achse) 5D (6 m) und in Längsrichtung (Y-Achse) 10D (12 m). Der Pfeil der Y-Achse gibt die Hauptwindrichtung an

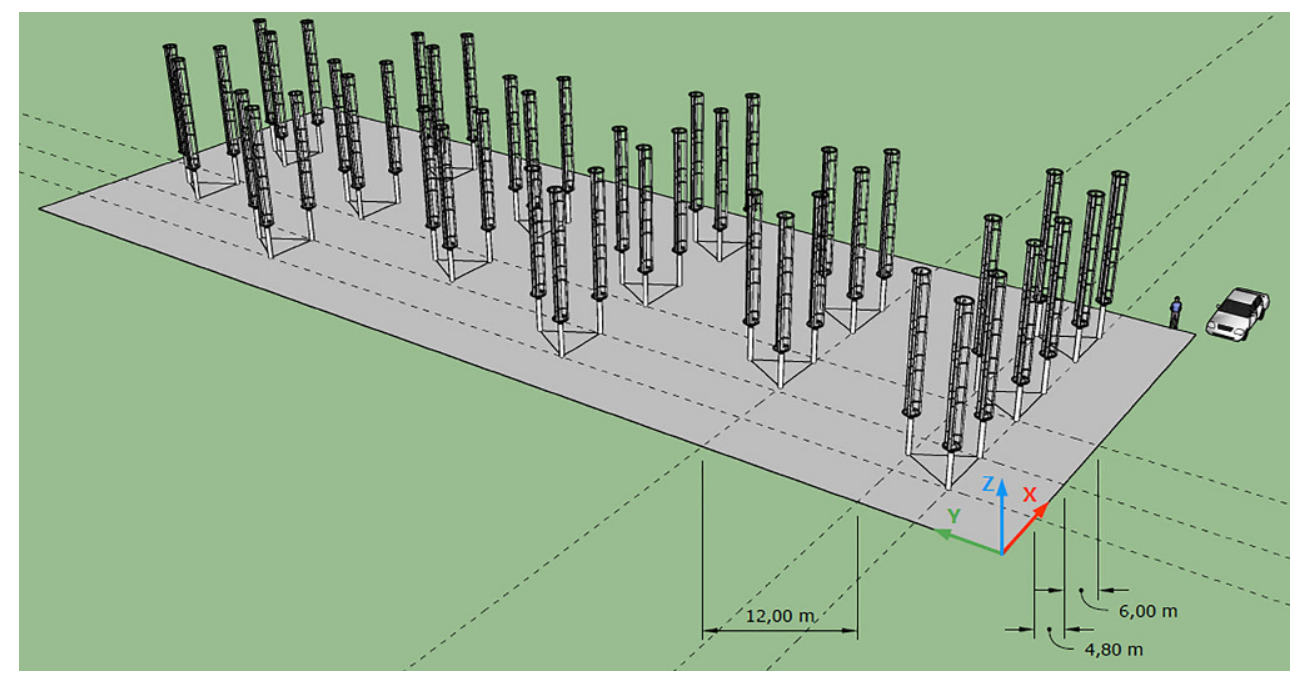

beiden Anlagentypen in einer Windparkkonfiguration sogar noch größer (Dabiri 2014). Die Berechnungen die in Abb. 5 dargestellt sind, weichen zum Teil signifikant von den Messungen in Abb. 6 ab. Grund für die unterschiedlichen Energiedichten ist, dass das bemessene Testfeld von Dabiri 2014 dem Schema der VAWT-Paare entsprach (vgl. Abb. 3 und 4). Die eigenen Berechnungen hingegen beziehen sich auf eine einzelne Anlage. Zudem könnte die Luftdichte zwischen den Messungen und der Berechnung abweichen, da sie temperaturabhängig ist. Hezaveh et al. haben 2017 anstatt der Anordnung von VAWTs in Form von Paaren (Dabiri 2011; Brownstein et al. 2016) anhand von Computersimulationen ein Array mit triangulären Clustern getestet (Hezaveh et al. 2018), welches in Abb. 7 als Schaubild zu erkennen ist. Es wurden verschiedene Abstände zwischen den Einzelanlagen sowie zwischen den triangulären Clustern hinsichtlich ihrer Auswirkungen auf den Energieoutput getestet. Zudem wurden diese mit den klassischen Windparkanordnungen bei konventionellen HAWTs, die sehr häufig linienförmig oder staffelförmig aufgebaut sind, verglichen. Es konnte festgestellt werden, dass die trianguläre Form die Strömungsbeschleunigungen am besten ausnutzt (Hezaveh et al. 2018).

Die Auswirkungen auf den Energieoutput wurden mit Abständen des 3-, 4- und 5-fachen Rotordurchmessers zwischen den Einzelanlagen in einem triangulären Cluster getestet, da diese die höchsten Wirkungsgrade zeigten. Der 5-fache Rotordurchmesser-Abstand wies, bezogen auf alle Windrichtungen sowie die Distanzen der Strömungswiederherstellung, die besten Werte auf. Insgesamt konnte mit dieser Ausrichtung 10\% mehr Energie erzeugt werden als bei drei isolierten Einzelanlagen. Schlussendlich hielten Hezaveh et al. fest, dass die trianguläre Clusterform die effektivste Array-Form ist, da sie die höchsten Wirkungsgrade und den geringsten Flächenverbrauch pro Turbine aufweist. Des Weiteren fanden sie heraus, dass nicht nur die Turbulenz- interaktionen zwischen einzelnen Anlagen innerhalb eines triangulären Clusters positiv sind, sondern auch die Turbulenzinteraktionen zwischen den verschiedenen triangulären Clustern.

Aufgrund der höheren Energiedichte, könnte ein entsprechend designter VAWT- Windpark einen gleich großen konventionellen Windpark daher an Gesamtleistung übertreffen, obwohl die VAWTs als Einzelanlage deutlich kleinere Leistungen und Wirkungsgrade aufweisen. Sowohl Laborals auch die Feldstudien haben gezeigt, dass ein Array in einer sogenannten „Truss Shape“ ${ }^{\text {"2 }}$ die beste Konfiguration für ein Cluster mit VAWTs darstellt (Brownstein et al. 2016). Ein weiterer Vorteil der VAWTs ist, dass sie aufgrund der kleineren Anlagengröße an Stellen errichtet werden können, an denen die größeren HAWT- Systeme aufgrund ihrer optisch bedrängenden Wirkung und anderer Faktoren keine Baugenehmigung erhalten würden. In einem weiteren Ansatz wird vorgeschlagen, die kleineren VAWTs in bereits bestehende, konventionelle Windparks zu implementieren. So könnten ungenutzte Flächen zwischen den HAWTs für die Energieproduktion erschlossen werden (Dabiri et al. 2015; AIP 2017).

Im letzten Jahr konnten Pagnini et al. zeigen, dass die Energieproduktion der VAWTs zudem stark von der lokalen Orographie und den lokalen Windverhältnissen, insbesondere der Turbulenzintensität, abhängt. Diese Faktoren sind bei der Abschätzung der Gesamtperformance eines VAWTWindparks wichtig (Pagnini et al. 2018).

\section{Akustische Signatur von VAWTs}

Wie in der Einleitung erwähnt, gehört neben dem Flächenbedarf der akustische Fußabdruck der konventionellen

\footnotetext{
${ }^{2}$ Die sogenannte „Truss Shape“ entspricht einer v-Form.
} 
HAWTs zu den größten Problemen beim Ausbau des Windenergiesektors. VAWTs hingegen, könnten laut Literatur auch in dieser Hinsicht deutliche Vorteile aufweisen (Dabiri 2011; Dabiri et al. 2015; Islam et al. 2013). Allerdings ist die Akustik dieser Anlagenform lediglich im Bereich der Geräuschmechanismen und der Schallemissionen in der unmittelbaren Umgebung einer Anlage durch computerbasierte Studien erforscht worden. Das Potential der VAWTs im Vergleich zu HAWTs im Bezug auf die Ausbreitung des Schalls ist bisher hingegen kaum erforscht. Es ist wichtig zu erwähnen, dass aufgrund der Komplexität des Themas nur auf die Grundlagen der Akustik im planerischen Kontext eingegangen wird. Folgend einige vertiefende Studien, die sich ausführlicher der Aeroakustik beschäftigen: Dessoky et al. 2019; Ghasemian and Nejat 2015; Tadamasa and Zangeneh 2011.

Bei der Energiegewinnung durch Windenergieanlagen entstehen zwei verschiedene Arten von Lärm: der mechanische und der aerodynamische Lärm. Mechanischer Lärm wird durch verschiedene Anlagenkomponenten der VAWTs, wie z.B. den Generator oder das Getriebe produziert. Diese Geräusche können entweder tonal oder breitbandig sein. Die Hersteller sind jedoch in der Lage diese Lärmquelle zu behandeln und einzudämmen. Der aerodynamische Lärm hingegen, ist die primäre Lärmquelle von VAWTs und wird daher zunächst genauer betrachtet.

Der zweite Teil dieses Unterkapitels beschäftigt sich dann mit der Ausbreitung des Lärms in der Atmosphäre. Mithilfe des in Deutschland etablierten Ausbreitungsmodells für Lärm von Windenergieanlagen in der freien Atmosphäre, wird anschließend beispielhaft berechnet, wie sich die Ausbreitung des Lärms von HAWTs und VAWTs unterscheidet.

\subsection{Aerodynamische Lärmquellen und - arten bei VAWTs}

Aerodynamischer Lärm, die primäre Lärmquelle von VAWTs, entsteht bei der Rotation der Rotoren durch die Luft und die entstehenden Turbulenzen in der Luftströmung. Dabei wird zwischen verschiedenen Lärmarten unterschieden, die im Folgenden aufgeführt werden (Botha et al. 2016; Pearson 2013).

\subsubsection{Trailing Edge Noise}

Wenn eine turbulente Strömung, die um eine Tragfläche strömt, die hintere Kante des Rotors („Trailing Edge“) überschreitet, werden die daraus resultierenden Druckschwankungen als Geräusch gestreut. Es wird ein Breitband-Geräusch erzeugt, das die größte Lärmquelle bei HAWTs darstellt und auch bei VAWTs eine große Rolle spielt. Die Lärmintensität hängt mit der Neigung, mit der die Strö- mung auf die Hinterkante trifft, zusammen. Grundsätzlich nimmt sie bei einer Reduzierung des Winkels zwischen der Strömung und der Hinterkante ab (Oerlemans et al. 2007; Pearson 2013).

\subsubsection{Laminar Boundary Layer Tonal Noise}

Der „Laminar Boundary Layer Tonal Noise“ wird als tonales Geräusch emittiert und entsteht, wenn eine laminare Strömung über die Hinterkante des Rotors strömt. Ein tonales Geräusch ist ein Ton mit schmaler Frequenz, der gegenüber dem Breitbandrauschen heraussticht. Dieser Ton benötigt, im Gegensatz zum umgebenden Breitbandrauschen, nicht viel Energie, um vom Menschen gehört zu werden. Man geht davon aus, dass das tonale Geräusch aus einem Rückkopplungseffekt entsteht, bei dem die Frequenz der Tollmien-Schlichting-Wellen ${ }^{3}$ in der laminaren Grenzschicht der Tragfläche beeinflusst werden. Dadurch können bestimmte Komponenten der Frequenz in der Strömung verstärkt werden. Die Akustikwellen breiten sich von einem Punkt, der stromabwärts des Rotors liegt, aus (siehe Abb. 8) (Arbey and Bataille 1983; Pearson 2013).

Der tonale Lärm wird von der Druckfläche emittiert (vgl. Abb. 8). Carr et al. und Pearson konnten zeigen, dass tonaler Lärm auch noch bei sehr hohen Reynoldszahlen ${ }^{4}$ emittiert wird, sofern der Anstellwinkel erhöht wird (Carr et al. 1971; Pearson 2013).

\subsubsection{Aerofoil Self Noise}

Diese Lärmart entsteht bei der Interaktion einer Tragfläche mit den Verwirbelungen in der eigenen Grenzschicht und mit den Verwirbelungen in der Nähe der Tragfläche. Brooks, Pope und Marcolini entwickelten 1989 dafür das maßgebliche empirische Rechenmodell und haben dabei die Interaktionen des sog. „Aerofoil Self Noise“ in fünf Kategorien unterschieden (Brooks et al. 1989):

1. Turbulent Boundary Layer Trailing Edge Noise,

2. Separation/Stall Noise,

3. Laminar Boundary Layer Vortex Shedding Noise,

4. Trailing Edge Bluntness Vortex Shedding Noise,

5. Tip Vortex Formation Noise.

\footnotetext{
${ }^{3}$ Begriff aus der Strömungslehre: Wenn Wind einen Körper umströmt entsteht direkt an der Oberfläche eine Grenzschicht. Steigt der Reibungswiderstand können Störungen an dieser Grenzschicht entstehen. Diese kleinen Störungen mit Wellencharakter werden als TollmienSchlichting-Wellen bezeichnet.

${ }^{4}$ Die Reynoldszahl beschreibt das Verhältnis der Trägheitskräfte und der Zahigkeitskräfte (Reibungskräfte) die wirken, wenn Luft um einen Körper strömt. Daraus kann man unter anderem ableiten, ob eine laminare oder eine kritische Strömung vorherrscht.
} 
Abb. 8 Entstehung von Laminar Boundary Layer Tonal Noise (Eigene Darstellung). Schema des Mechanismus durch den der Laminar Boundary Layer Tonal Noise entsteht

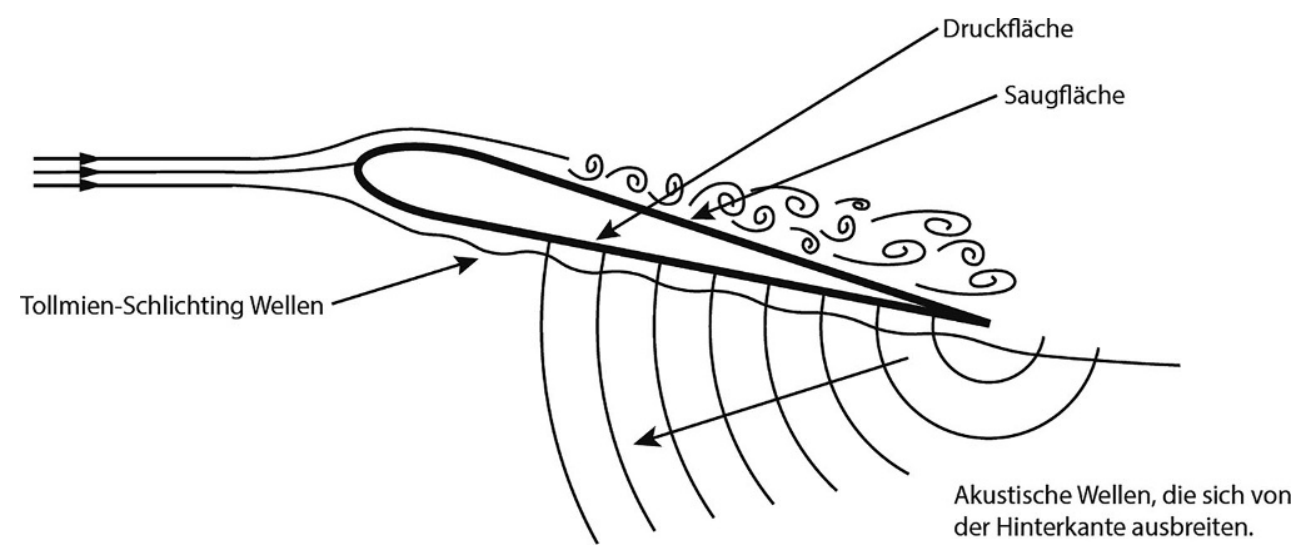

Die Dicke der Grenzschicht ist ein wichtiger Parameter, um diese Art von Lärm bestimmen zu können. Weitere Einflüsse, die zur Berechnung benötigt werden, sind die Machzahl der ungestörten Luftströmung, der Anstellwinkel und die geometrischen Parameter der Tragfläche. Der ,Separation/ Stall Noise" kann bei niedrigen Anstellwinkeln bis zu $10 \mathrm{~dB}$ lauter sein als der Lärmpegel des ,Turbulent Boundary Layer Trailing Edge Noise“. Bei moderaten Anstellwinkeln entsteht der Großteil des Lärms durch das Passieren von turbulenter, abgetrennter Strömung über die Hinterkante. Bei höheren Anstellwinkeln emittiert dann die gesamte Tragflächensehne den Lärm. (Brooks et al. 1989; Pearson 2013).

Im Gegensatz zu HAWTs, bei denen die „Trailing Edge" des Turbinenblattes die größte Quelle aerodynamischen Lärms ist (Oerlemans et al. 2007), konnte der Hauptmechanismus der aerodynamischen Lärmentstehung bei VAWTs bisher nicht festgelegt werden. Da die Luftströmung entlang des Rotorblattes einer VAWT grundsätzlich instationär ist, unterscheidet sich die Aerodynamik zwischen HAWTs und VAWTs wesentlich. Pearson 2013 vermutet, dass der "Seperation/ Stall noise“ bei niedrigen Schnelllaufzahlen und der „Trailing Edge Noise“ bei hohen Rotorblattgeschwindigkeiten signifikant an der Lärmentstehung beteiligt sind.

\subsection{Ausbreitung von Schall in der Atmosphäre}

In der freien Atmosphäre verteilt sich die Schallleistung mit zunehmendem Abstand auf eine immer größer werdende Kugeloberfläche, was zu einer kontinuierlichen Verringerung des Schalldruckpegels führt. Der vom Emittenten ausgesandte Schall wird als Schallleistungspegel bezeichnet, während der am Aufpunkt auftretende Schall Schalldruckpegel genannt wird. Mehrere atmosphärische Faktoren haben Einfluss auf die Ausbreitung der Schallleistung die in Tab. 1 aufgelistet sind.

Des Weiteren spielen auch die Windrichtung, die Luftfeuchtigkeit und die Lufttemperatur bei der Verstärkung oder Abminderung der Schallintensität eine Rolle, die jedoch in den etablierten Ausbreitungsmodellen sowie auch in den anschließenden Berechnungen nicht berücksichtigt werden.

\subsubsection{Schallimissionsprognosen bei Windenergieanlagen}

Das derzeit in Deutschland etablierte Ausbreitungsmodell für Schall von Windenergieanlagen basiert auf den Vorgaben der DIN ISO 9613-2 (DIN 2015). Anlagen mit einer Nabenhöhe $<30 \mathrm{~m}$ sind anhand des alternativen Verfahrens des ISO 9613-2 zu berechnen. Windenergieanlagen mit einer Nabenhöhe $>30 \mathrm{~m}$ unterliegen hingegen dem Interimsverfahren. Das Interimsverfahren ist eine überarbeitete Version des alten Verfahrens der ISO 9613-2, es befindet sich allerdings noch in der Entwicklung. In diesem Verfahren soll die Bodendämpfung, die eine Lärmreduktion von 4,8 dB ausmachen kann, aus der Berechnung herausfallen, da diese bei großen Anlagen kaum Auswirkungen auf die Schallausbreitung hat (DIN 2015). Bei kleinen Anlagen spielt sie jedoch eine größere Rolle und bleibt beim Schallprognoseverfahren enthalten. Die Abschirmungswirkung, d. h. die Reduzierung des Schalls durch Hindernisse (z.B. Gebäude) zwischen Anlage und Empfänger, wird nicht im etablierten Schallprognoseverfahren berücksichtigt, da sie für Anlagen mit großen Bauhöhen kaum eine Rolle spielen. Bei kleinen VAWTs hingegen, könnte die Abschirmungswirkung jedoch zu einer signifikanten Reduktion des Schalls führen (Agatz 2018; BWE 2019b).

Tab. 1 Atmosphärische Einflussfaktoren (DIN 2015)

\begin{tabular}{ll}
\hline $\mathrm{A}_{\text {div }}$ & Dämpfung aufgrund geometrischer Ausbreitung \\
$\mathrm{A}_{\mathrm{atm}}$ & Dämpfung aufgrund von Luftabsorption \\
$\mathrm{A}_{\mathrm{gr}}$ & Dämpfung aufgrund des Bodeneffekts \\
$\mathrm{A}_{\text {bar }}$ & $\begin{array}{l}\text { Dämpfung aufgrund Dämpfung aufgrund } \\
\text { von Abschirmung }\end{array}$ \\
& Dämpfung aufgrund verschiedener anderer Effekte \\
\hline
\end{tabular}


Die meisten Modelle der Kleinwindenergieanlagen (KWEAs) gehören zu der Kategorie der Stall-Anlagen. Stall-gesteuerte Windenergieanlagen können sich im Gegensatz zu den sogenannten Pitch-Anlagen nicht aus dem Wind drehen, sodass die Windströmung bei weiter ansteigender Windgeschwindigkeit an den Blattspitzen abreißt und es zum sogenannten „Stall-Effekt" kommt. Die Leistung kann in diesem Strömungszustand bei zunehmenden Windgeschwindigkeiten ab einem bestimmten Punkt nicht weiter ansteigen, die Schallemission erhöhen sich hingegen weiter. In niedrigen Höhen herrschen oft kleinere Windgeschwindigkeiten, weshalb es bei KWEAs selten zum Stall-Effekt kommt. Der Lärm liegt so häufig unter den Hintergrundgeräuschkulissen, die das Anlagengeräusch maskieren ${ }^{5}$. Daher spielen diese Anlagen in der Überwachungspraxis der Immissionsschutzbehörden bisher kaum eine Rolle. Aus diesem Grund gibt es auch keine speziellen Regelungen oder Hinweise in den schalltechnischen Messnormen für Kleinwindanlagen (Agatz 2018).

\subsubsection{Schallausbreitung in Windparks}

In dem etablierten Ausbreitungsmodell gilt für die Schallausbreitung einer Einzelanlage jederzeit die Mitwindbedingung ${ }^{6}$. Zudem wird vom Betrieb der Anlage unter Nennlast ausgegangen. Das dient dazu, den lautmöglichsten Zustand einer Anlage zu simulieren, damit eine hohe Planungssicherheit gewährleistet wird. Allerdings ist in Windparkkonfigurationen durch die entstehenden Turbulenzen nie für jede Windkraftanlage die Mitwindbedingung erfüllt. Zudem beeinflussen die Anlagen, die zuerst vom Windstrom getroffen werden, die Windgeschwindigkeiten stromabwärts. Dies hat negative Auswirkungen auf den Energieoutput und führt dazu, dass der Großteil der Windenergieanlagen eines Windparks ihre Nennleistung nicht erreichen kann (Agatz 2018). Beides führt zu einer Dämpfung der Schallimmissionen, die in den etablierten Ausbreitungsmodellen nicht berücksichtigt wird. Des Weiteren wird sowohl in den beschriebenen Rechenmodellen, als auch in den Berechnungen dieses Artikels, ausschließlich eine Einzelanlage betrachtet. In Windparks mit einer Vielzahl von Anlagen entsteht jedoch zusätzlich noch Interaktionslärm. Dieser ist von der Anzahl der Anlagen und den Abständen zwischen benachbarten Anlagen abhängig und erhöht die akustische Signatur einer Windenergieanlage.

\footnotetext{
5 Überlagerung des Anlagenlärms durch Umgebungsgeräusche.

${ }^{6}$ Die Mitwindbedingung beschreibt, dass Schall sich in alle Richtungen von der Schallquelle zum Immissionsort mit dem Wind (sozusagen mit Rückenwind) ausbreitet. Dieser theoretische Zustand dient dazu, den lautmöglichsten Zustand einer Anlage in jede Richtung zu simulieren, damit eine hohe Planungssicherheit gewährleistet wird.
}

\section{Vergleich der Schallausbreitung einer HAWT und einer VAWT}

In diesem Abschnitt wird anhand einer überschlägigen Berechnung die Ausbreitung des Schalls für eine HAWT und für eine VAWT abgeschätzt und miteinander verglichen. Zudem werden die Ausbreitungsszenarien den derzeit in Deutschland gültigen Grenzwerten für Lärm in Siedlungen gegenübergesellt.

Sowohl für die Schallprognose der HAWT, als auch für die der VAWT wurde das in Deutschland etablierte Ausbreitungsmodell nach der Norm DIN ISO 9613-2 (DIN 2015, S.6) in den Berechnungen verwendet. Die Formel für den sog. energieäquivalenten Oktavband-Dauerschalldruckpegel $\left[\mathrm{L}_{\mathrm{fT}}(\mathrm{DW})\right]$ lautet

$L_{\mathrm{fT}}(D W)=L W+D C-A$.

Parameter LW bezeichnet den Schalleistungspegel in Dezibel, für den in dieser Berechnung bei der HAWT $105 \mathrm{~dB}$ angegeben wurden. Dieser Wert wurde gewählt, da er dem Durchschnittswert von horizontalen Windenergieanlagen in Deutschland entspricht (BWE 2018). Bei der VAWT wurde ein Wert von $57,9 \mathrm{~dB}$ verwendet, welcher vom Turbinenmodell „QR5“ stammt (Botha 2015). In diesem Fall wurde der Wert eines anderen VAWT-Modells verwendet, da es für das Modell „Windspire 1,2kW turbine“ vom Hersteller keine Angabe zum Parameter LW gibt ${ }^{7}$. Bei dem Turbinenmodell „QR5“ handelt es sich, anders als bei der „Windspire 1,2 kW turbine“, um eine VAWT des klassischen DarrieusTyps. Die drei Rotorblätter dieses Modells sind nicht gerade wie bei einem H-Rotor (vgl. Abb. 4), sondern entsprechen einer Helixform. Der Lärmpegel von $57,9 \mathrm{~dB}$ ist im Vergleich zu anderen VAWT-Modellen relativ hoch. Dieser wurde jedoch gewählt, um einen verhältnismäßig lauten Zustand zu simulieren, damit im Falle eines Planungsvorhabens eine hohe Planungssicherheit gewährleistet wird. Parameter DC gibt die Richtwirkungskorrektur in Dezibel an, die beschreibt, um wieviel Einheiten der von der Punktquelle erzeugte, äquivalente Dauerschalldruckpegel in der festgelegten Richtung von dem Pegel einer angerichteten Punktschallquelle abweicht. Für eine ungerichtet, ins Freie abstrahlende Punktschallquelle, also auch für Windenergieanlagen, nimmt dieser Wert allerdings $0 \mathrm{~dB}$ an (Umweltbundesamt 2015). Parameter A beschreibt die Dämpfung in $\mathrm{dB}$, die während der Schallausbreitung zwischen Emit-

\footnotetext{
7 Windspire 2019 gibt lediglich an, dass die Anlage bei einem Abstand von $12 \mathrm{~m}$ einen Lärmpegel von $20 \mathrm{~dB}$ aufweist. Diese Angabe ist für die Berechnung in diesem Artikel allerdings unbrauchbar, da in dem Rechenmodell die Grundlautstärke benötigt wird.
} 


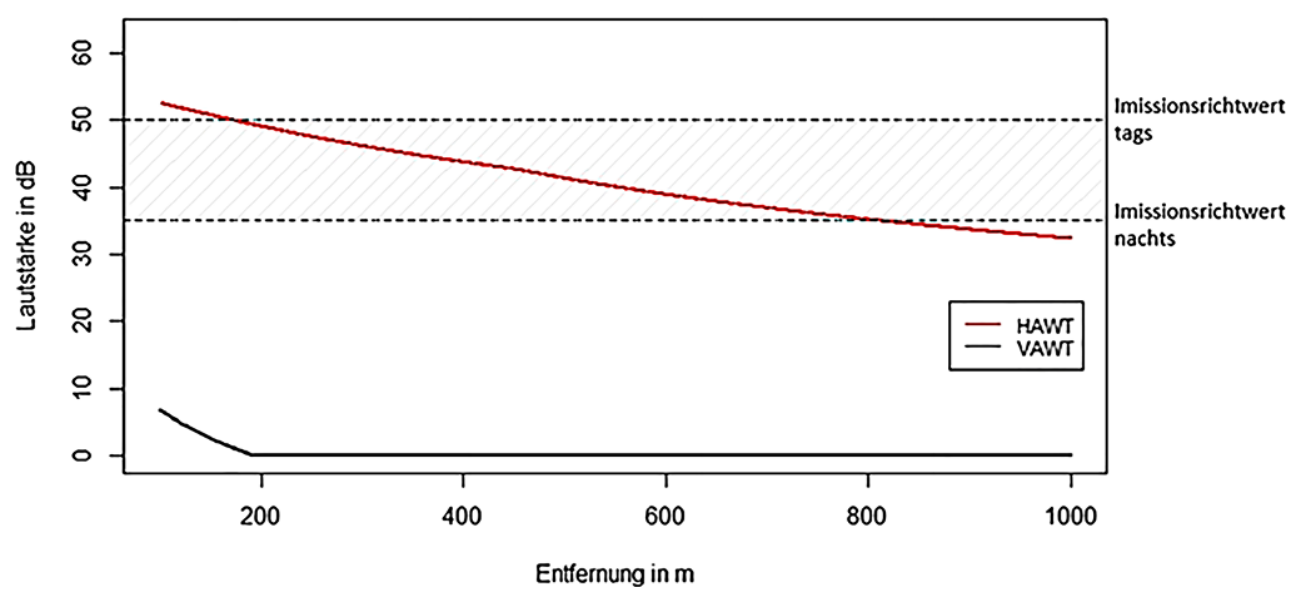

Abb. 9 Vergleich der Schallausbreitung (Eigene Darstellung). Die Grafik vergleicht die Schallausbreitung einer einzelnen HAWT und einer einzelnen VAWT bei einer Distanz von 100-1000 m zu der jeweiligen Turbine. Die beiden gestrichelten Linien stellen die Imissionsrichtwerte für Lärm bei Tag $(50 \mathrm{~dB})$ und bei Nacht $(35 \mathrm{~dB})$ in einem reinen Wohngebiet dar (LfU 2018). Laut Berechnungen muss eine Distanz von 812 $\mathrm{m}$ zwischen der HAWT und einem Wohngebiet liegen, damit die Anlage den rechtlichen Richtwerten für Lärm entspricht. Die VAWT hingegen unterschreitet bereits bei einer Distanz von 193 m 0 dB und wäre somit ab dieser Entfernung für das menschliche Gehör nicht mehr wahrnehmbar

Tab. 2 Ausschnitt der Ergebnisse von der Berechnung der atmosphärischen Dämpfungsterme $\mathrm{A}_{\mathrm{div}}, \mathrm{A}_{\mathrm{gr}}$ und $\mathrm{A}_{\text {atm }}$ auf eine bestimmte Entfernung zur Anlage für beide Anlagentypen

\begin{tabular}{lllllll}
\hline $\begin{array}{l}\text { Entfernung } \\
\text { zur Anlage }\end{array}$ & $\mathbf{A}_{\text {div }}($ HAWT $)$ & $\mathbf{A}_{\text {div }}($ VAWT $)$ & $\mathbf{A}_{\text {gr }}($ HAWT $)$ & $\mathbf{A}_{\text {gr }}($ VAWT $)$ & $\mathbf{A}_{\text {atm }}($ HAWT $)$ & $\mathbf{A}_{\text {atm }}($ VAWT $)$ \\
\hline $474 \mathrm{~m}$ & $64,913 \mathrm{~dB}$ & $64,499 \mathrm{~dB}$ & $0 \mathrm{~dB}$ & $4,762 \mathrm{~dB}$ & $0,992 \mathrm{~dB}$ & $0,946 \mathrm{~dB}$ \\
$475 \mathrm{~m}$ & $64,930 \mathrm{~dB}$ & $64,517 \mathrm{~dB}$ & $0 \mathrm{~dB}$ & $4,762 \mathrm{~dB}$ & $0,994 \mathrm{~dB}$ & $0,948 \mathrm{~dB}$ \\
$476 \mathrm{~m}$ & $64,946 \mathrm{~dB}$ & $64,535 \mathrm{~dB}$ & $0 \mathrm{~dB}$ & $4,762 \mathrm{~dB}$ & $0,996 \mathrm{~dB}$ & $0,950 \mathrm{~dB}$ \\
$477 \mathrm{~m}$ & $64,963 \mathrm{~dB}$ & $64,554 \mathrm{~dB}$ & $0 \mathrm{~dB}$ & $4,762 \mathrm{~dB}$ & $0,998 \mathrm{~dB}$ & $0,952 \mathrm{~dB}$ \\
$478 \mathrm{~m}$ & $64,979 \mathrm{~dB}$ & $64,572 \mathrm{~dB}$ & $0 \mathrm{~dB}$ & $4,763 \mathrm{~dB}$ & $1,000 \mathrm{~dB}$ & $0,954 \mathrm{~dB}$ \\
$479 \mathrm{~m}$ & $64,996 \mathrm{~dB}$ & $64,590 \mathrm{~dB}$ & $0 \mathrm{~dB}$ & $4,763 \mathrm{~dB}$ & $1,001 \mathrm{~dB}$ & $0,956 \mathrm{~dB}$ \\
\hline
\end{tabular}

tenten und Empfänger vorliegt. Term A in Gleichung (1) ist durch Gleichung (2) gegeben.

$A=A_{\text {div }}+A_{\text {atm }}+A_{\text {gr }}+A_{\text {bar }}+A_{\text {misc }}$

Die einzelnen Terme der Gleichung (2) beschreiben unterschiedliche atmosphärische Dämpfungseinflüsse, die bereits in Tab. 1 genannt wurden. In Tab. 2 werden einige Ergebnisse der Berechnung der atmosphärischen Dämpfungsterme $A_{\text {div }}, A_{g r}$ und $A_{a t m}$ gezeigt. Die Werte der Terme $A_{\text {div }}$ und $A_{a t m}$ liegen bei beiden Anlagentypen sehr dicht beieinander. Bei der Betrachtung der Werte des Parameters $\mathrm{A}_{\text {agr }}$ wird auffällig, dass die Dämpfung der VAWT, die der HAWT signifikant übersteigt. Wie vorher bereits erwähnt, werden die Parameter $\mathrm{A}_{\text {bar }}$ und $\mathrm{A}_{\text {misc }}$ in Schallimmissionsprognosen für Windenergieanlagen vernachlässigt und sind in Tab. 2 daher nicht abgebildet.

Neben dem durchschnittlichen Schallleistungspegel (LW) fließt auch die Nabenhöhe der jeweiligen Anlage mit in die Berechnung ein. Für sie wurde bei der HAWT ein Durchschnittswert von 128 m verwendet (Weston 2018). Im Falle der VAWT wurde ein Wert von $9,1 \mathrm{~m}$ gewählt, da dieser Wert der Bauhöhe des VAWT-Typs „Windspi- re 1,2kW turbine" entspricht (Windspire 2019). Weitere Anlagendaten fließen nicht in die Berechnungen ein.

Anhand Abb. 9 ist zu erkennen, dass sich die Ausbreitung der Schallemissionen der beiden Anlagentypen stark unterscheidet. In der Regel werden Schalldruckpegel von weniger als $35 \mathrm{~dB}$ nicht in Schallimissionsprognosen berücksichtigt, da darunter liegende Werte in der Überwachungspraxis der Schutzbehörden irrelevant sind. In Abb. 10 werden zum besseren Verständnis und zur Vergleichbarkeit einige alltägliche Beispiele zur Wahrnehmung und Wirkung von Schalldruckpegeln gezeigt. Der Wertebereich der Ordinate reicht in Abb. 9 jedoch bis $0 \mathrm{~dB}$, da der Schalldruckpegel von der VAWT in einem sehr niedrigen dB-Bereich liegt und ansonsten in der Abb. nicht darstellbar wäre. Bei der VAWT sinkt der Schalldruckpegel bereits bei etwa $200 \mathrm{~m}$ Entfernung auf $0 \mathrm{~dB}$ ab und ist somit für das menschliche Gehör nicht mehr wahrnehmbar. Bei der HAWT fällt dieser Wert bei der gleichen Entfernung auf $<50 \mathrm{~dB}$ und unterschreitet somit den Imissionsrichtwert für Schall bei Tag in reinen Wohngebieten (TA Lärm 2017) (vgl. obere gestrichelte Linie in Abb. 9). Damit eine HAWT genehmigungsfähig werden kann, darf sie den Imissionsrichtwert für Schall bei Nacht in reinen Wohnge- 


\section{Schallpegel in $\mathrm{dB}(\mathrm{A})$}

\begin{tabular}{|c|c|c|}
\hline Schmerzschwelle & 130 & Düsenjet \\
\hline \multirow{3}{*}{ Schädigungsbereich } & 120 & Livekonzert \\
\hline & 110 & Propellerflugzeug \\
\hline & 100 & HAWT (in $1 \mathrm{~m}$ Enternung) \\
\hline \multirow{3}{*}{ Belästigungsbereich } & 90 & Diskothek \\
\hline & 80 & Pkw- Vorbeifahrt \\
\hline & 70 & Rasenmäher \\
\hline \multirow{3}{*}{$\begin{array}{l}\text { Tagespegel im } \\
\text { Wohnbereich }\end{array}$} & 60 & Unterhaltung \\
\hline & 50 & Regen \\
\hline & 40 & Bibliothek \\
\hline \multirow{3}{*}{ Leiser Bereich } & 30 & Flüstern \\
\hline & 20 & Blätterrauschen \\
\hline & 10 & Schneefall \\
\hline Hörschwelle & 0 & \\
\hline
\end{tabular}

Abb. 10 Schallpegel (Eigene Darstellung). Die typische Wahrnehmung und Wirkung verschiedener Schalldruckpegel und einige alltägliche Beispiele. Eine horizontale Windenergieanlage in Deutschland hat in $1 \mathrm{~m}$ Entfernung im Durchschnitt einen Schalldruckpegel von $105 \mathrm{~dB}$ (BWE 2018)

bieten von $35 \mathrm{~dB}$ nicht überschreiten (untere gestrichtelte Linie in Abb. 9). Ausgehend von Abb. 9 muss die HAWT nach dem hier verwendeten Rechenmodell etwa $800 \mathrm{~m}$ vom Wohnungsgebiet entfernt sein, um diese Bedingung zu erfüllen. Aus der Literatur sind ähnliche Abstände bekannt (z.B. BWE 2018). Die VAWT hingegen, wäre bereits bei einem deutlich kleineren Abstand genehmigungsfähig. $\mathrm{Ab}$ welcher Distanz der Immissionsrichtwert bei Nacht genau unterschritten wird, ist aus Abb. 9 nicht ersichtlich, da für die Entfernung $<100 \mathrm{~m}$ mit dem Modell keine Werte berechnet werden können. Die verwendete ISO Methode ist lediglich für Distanzen von 100-1000 m zur Emissionsquelle anwendbar.

Möllerström et al. haben ähnliche Erkenntnisse gesammelt, die auch auf Vorteile der VAWTs im Hinblick auf den akustischen Fußabdruck hindeuten und somit die Ergebnisse der Imissionsprognose dieser Arbeit stützen. Sie untersuchten die Schallausbreitung einer HAWT und einer VAWT, die jeweils eine vergleichbare Bauhöhe und eine vergleichbare Nennleistung aufweisen. Dabei fanden sie heraus, dass die Grundlautstärke der VAWT um einige Dezibel leiser ist als bei HAWTs, obwohl bei beiden Messungen die gleichen Windgeschwindigkeiten herrschten (Möllerström et al. 2015). Zudem nimmt der Lärm bei der VAWT laut Untersuchungen stärker ab als bei den horizontalen Windkraftanlagen. Als Grund führen Möllerström et al. die unterschiedlichen Schallfrequenzen der beiden Anlagenarten auf. Bei VAWTs werden im Gegensatz zu HAWTs kaum niedrige Frequenzen emittiert. Diese niedrigen Frequenzen werden von der Atmosphäre bei der Schallausbreitung weniger absorbiert als höhere Frequenzen. Es kommt daher zu einer stärkeren Schalldämpfung bei VAWTs. Des Weiteren wird bei vertikalen Anlagen mehrheitlich Lärm emittiert, der ähnliche Frequenzen wie der Hindergrundlärm aufweist. Das könnte zu einer stärkeren Maskierung der Anlagengeräusche von VAWTs führen und sich positiv auf den akustischen Fußabdruck auswirken (Möllerström et al. 2014).

Trotz der Übereinstimmungen aus der Literatur hat das Ausbreitungsmodell einige Beschränkungen. Zum einen sind die Prognosewerte erst ab einer Entfernung von $>100 \mathrm{~m}$ und nur bis zu einem Abstand zur Emissionsquelle von $<1000 \mathrm{~m}$ gültig, weshalb sich die X-Achse in Abb. 9 auch auf diesen Bereich beschränkt. Es ist somit unklar, wie sich die Schallausbreitung $<100 \mathrm{~m}$ verhält und bei welchem Abstand die Imissionsrichtwerte einer VAWT bei Tag und bei Nacht bereits unterschritten werden. Des Weiteren sind die Berechnungen immer unabhängig von der Windgeschwindigkeit, da in dem Modell von der Mitwindbedingung und der Volllast der Anlagen ausgegangen wird.

\section{Fazit und Ausblick}

Die Untersuchungen dieser Arbeit legen nahe, dass die bisher wenig im Fokus der Forschung und Öffentlichkeit stehenden VAWTs ein hohes Zukunftspotenzial bieten. Im Vergleich zu den konventionellen HAWTs weisen sie einen kleineren Flächenverbrauch auf und haben zudem eine geringere akustische Signatur.

Der geringere Flächenverbauch ergibt sich aus einer höheren Energiedichte, die gegeben ist, wenn VAWTs in einer speziell ausgerichteten Windparkkonfiguration implementiert werden. Die entstehenden Turbulenzen bei der Durchströmung von Wind durch ein solches Array, können von den stromabwärts liegenden Anlagen effektiv zur Energiegewinnung genutzt werden. Da zudem eine schnelle energetische Wiederaufladung des Windes erfolgt, können die VAWTs deutlich näher zu den benachbarten Anlagen stehen, als es bei HAWTs der Fall ist. Somit kann laut Literatur in Windparks mit VAWTs bis zu zehn Mal mehr Energie pro Flächeneinheit erzeugt werden, als in konventionellen HAWT-Windparks (Dabiri 2011). Die eigenen Berechnungen dieser Arbeit zur erzielbaren Energiedichte von VAWTs liegen etwas unterhalb dieser Größenordnung. Dennoch deuten sie ebenfalls auf eine höhere Energieausbeute pro 
Flächeneinheit im Vergleich zu HAWT-Windparkkonfigurationen hin.

Die Schallemissionen von Windkraftanlagen setzen sich aus einer Vielzahl von Lärmarten zusammen und ihr Entstehungsprozess ist hochkomplex. Laut den Erkenntnissen aus der vorhandenen Literatur und aus den Berechnungen dieser Arbeit, weisen VAWTs eine geringere akustische Signatur als HAWTs auf. Sie ist maßgeblich auf die niedrigere Grundlautstärke zurückzuführen. Außerdem spielt das spezifische Ausbreitungsverhalten des Schalls bei diesen Anlagen eine Rolle. Die Bodendämpfung $\left(\mathrm{A}_{\mathrm{gr}}\right)$, die mit der Bauhöhe der jeweiligen Anlage zusammenhängt, hat einen größeren Einfluss auf die Schalldämpfung von VAWTs als bei HAWTs (vgl. Tab. 2). Durch die Schallimissionsprognose in dieser Arbeit konnte veranschaulicht werden, dass die VAWTs die Lärmrichtwerte für Wohngebiete bei deutlich kürzeren Distanzen unterschreiten als HAWTs. Dies würde nicht nur dazu führen, dass neue Räume und deutlich mehr Flächen für den Bau von Windenergieanlagen verwendet werden können. Weiterhin könnten VAWTs folglich das durch die Lärmemissionen entstehende Konfliktpotenzial und die dahingehenden Bedenken der Bevölkerung reduzieren und somit die gesellschaftliche Akzeptanz steigern.

Die durchgeführten Berechnungen weisen jedoch einige Beschränkungen auf. Daher muss das Rechenmodell in $\mathrm{Zu}-$ kunft an die speziellen Eigenschaften der VAWTs angepasst werden. Eine Berücksichtung der jeweiligen Rotationsgeschwindigkeit, der Schnelllaufzahl oder des Anstellwinkels, die sich bei VAWTs und HAWTs stark unterscheiden, könnte hierbei eine Möglichkeit sein. Zudem muss es mit dem weiterentwickelten Modell möglich sein, die Werte für die Distanz $<100 \mathrm{~m}$ der Schallausbreitung zu berechnen, was mit dem Modell, das in dieser Arbeit verwendet wurde, nicht realisierbar war. Des Weiteren wurde in den Berechnungen dieser Arbeit lediglich die Lärmemission für eine Einzelanlage beider Windturbinentypen berechnet. In der Realität würde in einem VAWT-Array jedoch eine Vielzahl von Anlagen Lärm emittieren. In einem solchen Windparkszenario wird die Gesamtschallemission durch die Addition der verschiedenen Schalldruckpegel im Vergleich zu den hier berechneten Werten deutlich höher sein.

Die Ergebnisse zeigen dennoch, dass VAWTs das Potenzial besitzen die negativen Effekte der konventionellen Windenergieanlagen zu vermeiden. Die geringere visuelle und akustische Signatur sowie der kleinere Flächenverbrauch dieser Anlagen, bieten im Vergleich zu den konventionellen HAWTs neue Flächennutzungsmöglichkeiten. Windparks mit VAWTs könnten beispielsweise auf ungenutzen Flächen in der Nähe von Siedlungen oder anderen Schutzgebieten installiert werden, auf denen HAWTs aufgrund der Überschreitung verschiedener Grenzwerte nicht zulässig sind. Dadurch könnten Probleme die beim Ausbau des Windenergiesektors bestehen, insbesondere Flä- chennutzungskonflikte, reduziert und eine erhöhte gesellschaftliche Akzeptanz erreicht werden.

Funding Open access funding provided by Max Planck Society.

Open Access Dieser Artikel wird unter der Creative Commons Namensnennung 4.0 International Lizenz (http://creativecommons.org/ licenses/by/4.0/deed.de) veröffentlicht, welche die Nutzung, Vervielfältigung, Bearbeitung, Verbreitung und Wiedergabe in jeglichem Medium und Format erlaubt, sofern Sie den/die ursprünglichen Autor(en) und die Quelle ordnungsgemäß nennen, einen Link zur Creative Commons Lizenz beifügen und angeben, ob Änderungen vorgenommen wurden.

\section{Literatur}

Agatz M (2018) Windenergie-Handbuch. http://windenergie-handbuch. de/wp-content/uploads/2019/02/Windenergie-Handbuch-2018. pdf. Zugegriffen: 29. Juli 2019

AIP (2017) Breakthrough research for testing and arranging vertical axis wind turbines. American Institute of Physics. https://phys. org/news/2017-02-breakthrough-vertical-axis-turbines.html. Zugegriffen: 25. Juli 2019

Arbey H, Bataille J (1983) Noise generated by airfoil profiles placed in a uniform laminar flow. J Fluid Mech 134(33):33-47. https://doi. org/10.1017/S0022112083003201

Betz A (1920) Das Maximum der theoretisch möglichen Ausnützung des Windes durch Windmotoren. Z Ges Turbinenwesen. https:// doi.org/10.1260/0309-524X.37.4.441

Botha J (2015) A novel method of vertical axis wind turbine noise prediction. https://www.conforg.fr/euronoise2015/proceedings/ data/articles/000363.pdf. Zugegriffen: 5. Aug. 2019 (EuroNoise 2015, Maastricht)

Botha JDM, Rasam A, Catháin D, Rice H, Shahrokhi A (2016) Some noise predictions for small wind turbines. Proceedings of ISMA, S 4019-4032

Brooks T, Pope S, Marcolini M (1989) Airfoil self-noise and prediction. NASA - Reference Publication 1218.

Brownstein I, Kinzel M, Dabiri JO (2016) Performance enhancement of downstream vertical-axis turbines. J Renew Sustain Energy. https://doi.org/10.1063/1.4964311

BWE (2018) Schallimmissionen von Windenergieanlagen. Bundesverband Windenergie. https://www.wind-energie.de/fileadmin/ redaktion/dokumente/publikationen-oeffentlich/themen/01mensch-und-umwelt/05-schall/20181123_BWE_ Informationspapier_Schall_und_WEA.pdf. Zugegriffen: 28. Juli 2019

BWE (2019a) Energie in Deutschland - Zahlen und Fakten. Bundesverband Windenergie. https://www.wind-energie.de/themen/ zahlen-und-fakten/deutschland/. Zugegriffen: 28. Juli 2019

BWE (2019b) Schall. Bundesverband Windenergie. https://www. wind-energie.de/themen/mensch-und-umwelt/schall/. Zugegriffen: 28. Juli 2019

Carr LW, McAlister KW, McCroskey WJ (1971) Analysis of the development of dynamic stall based on oscillating airfoil experiments. Ames Research Center and US Army Air Mobility Research and Development Laboratory, Moffett Field

Dabiri JO (2011) Potential order-of-magnitude enhancement of wind farm power density via counter-rotating vertical-axis wind turbine arrays. J Renew Sustain Energy. https://doi.org/10.1063/1. 3608170

Dabiri JO (2014) Emergent aerodynamics in wind farms. Phys Today 67(10):66-67. https://doi.org/10.1063/PT.3.2559

Dabiri JO (2017) Biomechanics. how fish feel the flow. Nature 547(7664):406-407. https://doi.org/10.1038/nature23096 
Dabiri JO, Greer JR, Koseff JR, Moin P, Peng J (2015) A new approach to wind energy: opportunities and challenges. 4th International Congress in Advances in Applied Physics and Materials Science, S 51-57

Dena (2010) Dena-Netzstudie II - Integration erneuerbarer Energien in die deutsche Stromversorgung im Zeitraum 2015-2020 mit Ausblick auf 2025. https://www.dena.de/fileadmin/user_upload/ Download/Dokumente/Studien__Umfragen/Endbericht_denaNetzstudie_II.PDF. Zugegriffen: 5. Aug. 2019

Dessoky A, Lutz T, Bangga G, Kraemer E (2019) Computational studies on darrieus vawt noise mechanisms employing a high order ddes model. Renew Energy 143:404-425. https://doi.org/10. 1016/j.renene.2019.04.133

DEWI (2015) Rotorblattspitze innerhalb oder außerhalb der Konzentrationszone: Welchen Einfluss hat dies auf den Flächenbedarf einer Windenergieanlage? https://www.dewi.de/dewi_res/ fileadmin/pdf/studies/DEWI_KSA_Vergleich_Flaechenbedarf_ WEA_Rotorblatt_innen_au\%C3\%9Fen.pdf. Zugegriffen: 3. Aug. 2019

DIN (2015) Dokumentation zur Schallausbreitung - Interimsverfahren für Windkraftanlagen, Fassung 2015-05.1. https://www.din. de/blob/187138/eb8abdf16f058490895.pdf. Zugegriffen: 3. Aug. 2019

Eriksson S, Bernhoff H, Leijon M (2008) Evaluation of different turbine concepts for wind power. Renewable and Sustainable Energy Reviews 12(5):1419-1434. https://doi.org/10.1016/j.rser.2006. 05.017

Fachagentur-Windenergie (2019a) Akzeptanz für die Windenergie. https://www.fachagentur-windenergie.de/themen/akzeptanz. html. Zugegriffen: 2. Aug. 2019

Fachagentur-Windenergie (2019b) Überblick zu den Abstandsempfehlungen zur Ausweisung von Windenergiegebieten in den Bundesländern. https://www.fachagentur-windenergie.de/fileadmin/ files/PlanungGenehmigung/FA_Wind_Abstandsempfehlungen_ Laender.pdf. Zugegriffen: 2. Aug. 2019

Fraunhofer (2019) Bisherige Entwicklung und Ausbauszenarien für die Windenergie on- und offshore. http://windmonitor.iee.fraunhofer. de/windmonitor_de/1_wind-im-strommix/1_energiewende-indeutschland/5_Ausbau_der_Windenergie/. Zugegriffen: 2. Aug. 2019

Gemmell BJ, Fogerson SM, Costello JH, Morgan JR, Dabiri JO, Colin SP (2016) How the bending kinematics of swimming lampreys build negative pressure fields for suction thrust. J Exp Biol 219(24):3884-3895. https://doi.org/10.1242/jeb.144642

Ghasemian M, Nejat A (2015) Aero-acoustics prediction of a vertical axis wind turbine using large eddy simulation and acoustic analogy. Energy 88:711-717. https://doi.org/10.1016/j.energy.2015.05. 098

Hezaveh SH, Bou-Zeld E, Dabiri JO, Kinzel M, Cortina G, Martinelli L (2018) Increasing the power production of vertical-axis wind-turbine farms using synergistic clustering. Boundary-Layer Meteorol 169(2):275-296. https://doi.org/10.1007/s10546-018-0368-0

Hübner G, Pohl J (2015) Mehr Abstand - mehr Akzeptanz ? Ein umweltpsychologischer Studienvergleich. FA Wind. https:// www.fachagentur-windenergie.de/fileadmin/files/Akzeptanz/FAWind_Abstand-Akzeptanz_Broschuere_2015.pdf. Zugegriffen: 3. Aug. 2019

Hui I, Cain BE, Dabiri JO (2018) Public receptiveness of vertical axis wind turbines. Energy Policy 112:258-271. https://doi.org/10. 1016/j.enpol.2017.10.028

IPCC (2018) Global Warming of 1.5 C. https://report.ipcc.ch/sr15/pdf/ sr15_spm_final.pdf. Zugegriffen: 14. Juli 2019

Islam M, Mekhilef S, Saidur R (2013) Progress and recent trends of wind energy technology. Energy Rev 21:456-468. https://doi.org/ 10.1016/j.rser.2013.01.007
Kinzel M, Araya DB, Dabiri JO (2015) Turbulence in vertical axis wind turbine canopies. Phys Fluids. https://doi.org/10.1063/1. 4935111

Lärm TA (2017) Abschnitt 6.1 TA Lärm - Sechste Allgemeine Verwaltungsvorschrift zum Bundes-Immissionsschutzgesetz. https:// www.jurion.de/gesetze/ta_laerm/6.1/. Zugegriffen: 14. Juli 2019

LfU (2018) Windenergie in Bayern. Bayerisches Landesamt für Umwelt. https://www.lfu.bayern.de/buerger/doc/uw_118_ windenergie_in_bayern.pdf. Zugegriffen: 14. Juli 2019

Möllerström E, Larsson S, Ottermo F, Hylander J, Baath L (2014) Noise propagation from a vertical axis wind turbine. https://www. acoustics.asn.au/conference_proceedings/INTERNOISE2014/ papers/p829.pdf. Zugegriffen: 24. Juli 2019 (Inter.noise 2014)

Möllerström E, Ottermo F, Hylander J, Bernhoff H (2015) Noise emission of a $200 \mathrm{kw}$ vertical axis wind turbine. Energies. https://doi. org/10.3390/en9010019

Oerlemans S, Sijtsma P, Mendez-Lopez B (2007) Location and quantication of noise sources on a wind turbine. J Sound Vib 299:4-5. https://doi.org/10.1016/j.jsv.2006.07.032

Pagnini L, Piccardo G, Repetto MP (2018) Full scale behavior of a small size vertical axis wind turbine. Renew Energy 127:41-55. https://doi.org/10.1016/j.renene.2018.04.032

Pearson C (2013) Vertical axis wind turbine acoustics. PhD thesis, Cambridge University Engineering Department. https:// www.repository.cam.ac.uk/bitstream/handle/1810/245256/Thesis \%20-\%20VAWT\%20Noise\%20-\%20Charlie\%20Pearson.pdf sequence $=1$. Zugegriffen: 22. Juli 2019

Rolin V, Porté-Agel F (2018) Experimental investigation of verticalaxis wind-turbine wakes in boundary layer flow. Renew Energy 118:1-13. https://doi.org/10.1016/j.renene.2017.10.105

Ryan KJ, Coletti F, Elkins CJ, Dabiri JKEJO (2016) Three-dimensional flow field around and downstream of a subscale model rotating vertical axis wind turbine. Exp Fluids. https://doi.org/10. 1007/s00348-016-2122-z

Stuttgart VG (2013) Optisch bedrängende wirkung einer windkraftanlage. Natur und Recht 35(11):836-840. https://doi.org/10.1007/ s10357-013-2553-8

Tadamasa A, Zangeneh M (2011) Numerical prediction of wind turbine noise. Renew Energy 36(7):1902-1912. https://doi.org/10.1016/j. renene.2010.11.036

Twardella D (2013) Bedeutung des Ausbaus der Windenergie für die menschliche Gesundheit. Umweltbundesamt. https://www. umweltbundesamt.de/sites/default/files/medien/360/publikationen/ ausbau_windenergie_s_14_19n.pdf. Zugegriffen: 29. Juli 2019

Umweltbundesamt (2015) Anleitung für die Modellbildung zur Schallimmissionsprognose nach ÖNORM ISO 9613-2: 2008 und ÖNORM EN 12354-4. http://www.umweltbundesamt.at/ fileadmin/site/umweltthemen/laerm/forum_schall/downloads/ Anleitung_zur_Modellbildung_2015.pdf. Zugegriffen: 29. Juli 2019

Umweltbundesamt (2018) Energiebedingte Emissionen. https://www. umweltbundesamt.de/daten/energie/energiebedingte-emissionen. Zugegriffen: 29. Juli 2019

Weston D (2018) Average turbine size reaches 2.4MW. https://www. windpowermonthly.com/article/1461367/average-turbine-sizereaches-24mw-updated. Zugegriffen: 29. Juli 2019

Windspire (2019) Windspire Specifications - Standard 1,2kW Unit. http://www.eciwas.com/Products/Wind_Products/Windspire/ WindspireSpecSheetDec09.pdf. Zugegriffen: 29. Juli 2019 